\title{
Intertrochanteric stress fracture following the Comrades Marathon
}

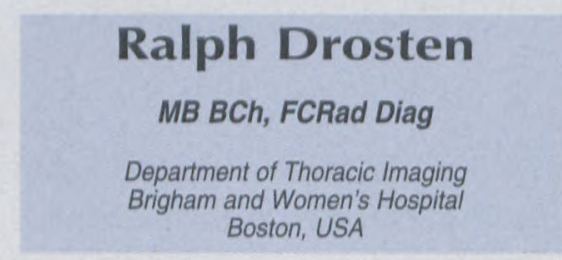

\section{Case presentation}

A 33-year-old man presented to the X-ray department complaining of right hip pain 3 days after completing the 2000 Comrades Marathon. He had experienced significant pain during the last third of the marathon.

The pelvic and right hip X-rays on the day of presentation (Fig. 1) demonstrated no visible fracture, sclerosis, medullary irregularity or effusions.
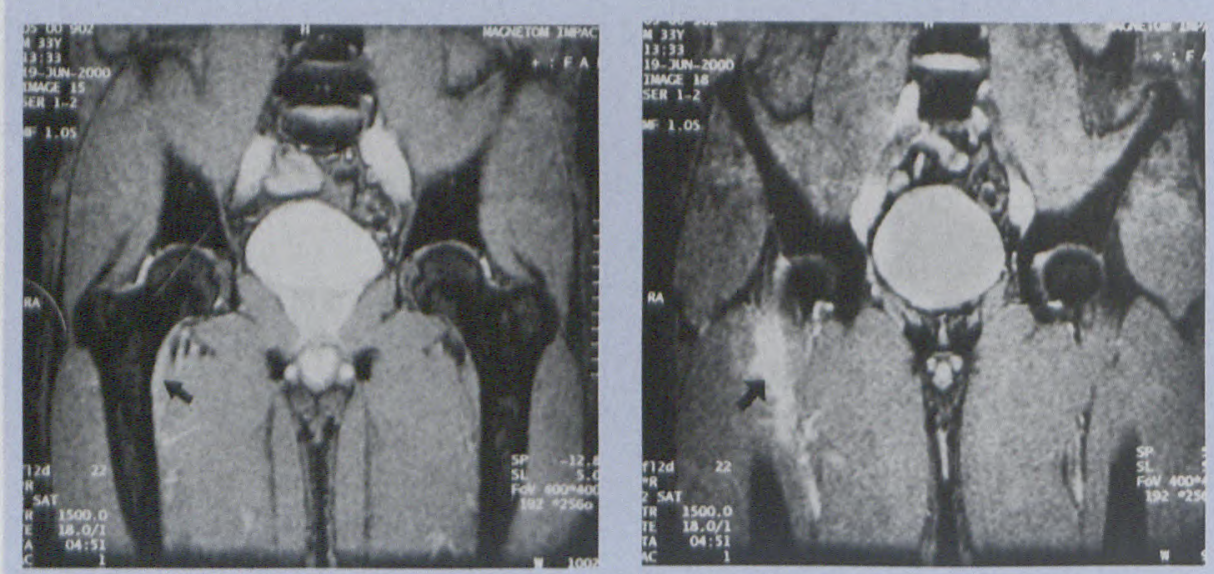

Figs 2 and 3 . Fluid suppression MRI of the right hip showing oedema in the vastus muscle group (arrow), but no intramedullary signal changes 3 days after the Comrades Marathon.
(Figs 2 and 3) confirmed the ultrasonographic findings. No cortical or medullary bone changes, fracture lines or asymmetry were detected, and no hip effusion was visible. Fluid was noted tracking medial to the proximal right femur. Oedema of the proximal vastus medialis and intermedialis muscles was confirmed.

The patient was treated conservatively. He presented 10 days later complaining of unremitting pain in the right hip. He had been for two short 'leg stretching' runs in the interim. Repeat X-rays of the right hip (Figs 4 and 5) demonstrated an obvious illdefined area of sclerosis traversing the right femoral intertrochanteric region - consistent with a stress fracture. No clearly defined lucent fracture line was noted.

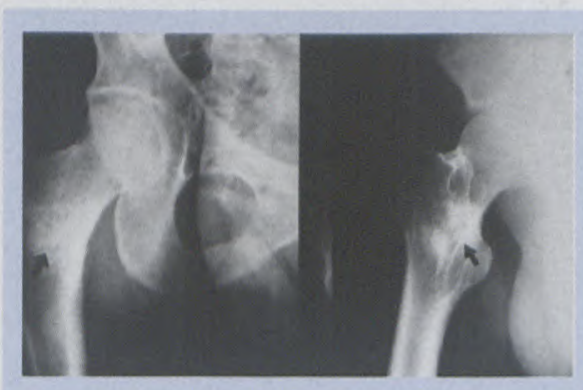

Figs 4 and 5. Right hip X-rays 10 days after the Comrades Marathon showing ill-defined sclerosis in the intertrochanteric region (arrows).

A ${ }^{99 m}$ Tc MDP isotope study was performed (Fig. 6). Prominent uptake in the right intertrochanteric region confirmed the X-ray findings of a right intertrochanteric stress fracture.

\section{Discussion}

Stress fractures occur as a result of chronic repetitive trauma to normal bone (abnormal stresses to normal bone). A stress fracture occurring in an otherwise normal bone as a result of normal activity is termed a fatigue fracture (normal stress to normal bone). A fracture occurring as a result 


\section{CASE REPORT}

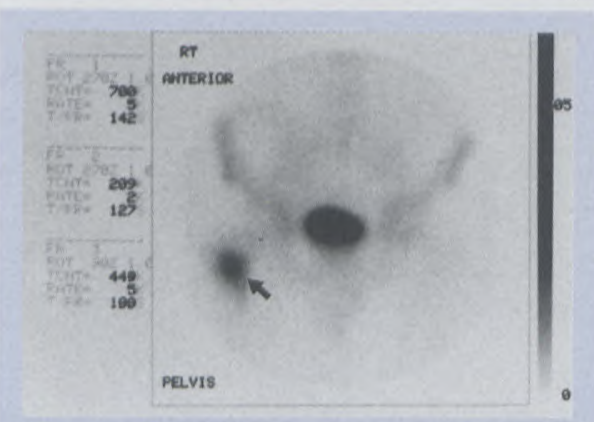

Figs 6. ${ }^{99 m} T C$ MDP isotope study 10 days after the Comrades Marathon demonstrating uptake in the right intertrochanteric region (arrow), consistent with a stress fracture.

of normal stresses applied to abnormal bone is an insufficiency fracture (normal stress to abnormal bone).

The repetitive trauma causing the stress fracture ultimately results in structural failure of the bone. ${ }^{1}$ Such injuries may be difficult to diagnose at the initial presentation. A subtle periosteal reaction or a transverse band of linear sclerosis may develop 2 - 3 weeks after the onset of symptoms. The fracture line, as in our example, is often never identified. Our patient's day $10 \mathrm{X}$-ray demonstrated sclerosis - perhaps secondary to the repeated trauma experienced during the post-marathon 'leg stretching' runs.

Common sites for stress fractures include the metatarsal shafts ('march fractures'), pubic rami, femoral neck, tibial and fibular shafts and the calcaneal tuberosity.

Indirect radiological signs are often useful in suggesting an acute fracture. Soft tissue swelling is usually present adjacent to the fracture site. Displacement or obliteration of normal fat pads can be a clue to acute fracture haematoma or joint distension. The presence of lipohaemoarthrosis is prima-facie evidence of an intra-articular fracture, and is best assessed using cross table $\mathrm{X}$-rays, i.e. a horizontal beam.

Isotope bone scans and MRI are very sensitive methods of detection of this type of injury, and may be positive at the time of initial presentation.

MRI is much more sensitive than conventional radiography in the detection of stress and occult fractures and will usually be diagnostic immediately after the injury. ${ }^{2}$ The absence of medullary marrow changes on our patient's day 3 MRI gives further credence to the possibility that the stress fracture developed, or extended, during the days after the marathon. Stress fractures produce a pattern of bone marrow oedema similar to that seen with acute trauma. A fracture line, if seen, is a persistent low signal band on all pulse sequences. ${ }^{3}$

Isotope scans are a sensitive modality in the assessment of a suspected stress fracture. With chronic stress, mild diffuse cortical thickening is often seen; however, a focus of increased uptake on one or other cortical margin indicates the presence of a stress microfracture. Within the first few hours following the injury there is a post-traumatic inflammatory reaction and the blood flow and blood pool images show hyperaemia around the site of injury. Within 24 hours early osteoblastic activity commences within the callus and the delayed scan will be positive. ${ }^{4}$ This repair may be retarded in patients with marked osteoporosis. For the next few weeks all three phases of the bone scan will be intensely hot, but eventually the increased blood flow returns to nor$\mathrm{mal}$. The hyperaemia of repair will be visible on the blood pool imaging as long as there is active callus formation. The hyperaemia of repair subsides during the late remodelling phase, or when non-union has occurred, but the delayed scan remains hot for several months, as long as remodelling continues.
Thus the age and severity of trauma can be estimated by observing the relative uptake in the three phases of the bone scan.

A bone scan is not recommended as the primary imaging modality in detecting fractures, but it is useful when the radiographs are negative despite symptoms, or to assess patients with multiple trauma. Fractures in radiologically elusive sites, such as the sacro-iliac (SI) joints, anterior ribs, sternum, base of skull, etc. may be visualised.

\section{Conclusion}

The stress fracture (sclerosis in the right intertrochanteric region) became radiologically evident by the tenth day. The absence of medullary marrow changes on our patient's day 3 MRI gives further credence to the possibility that the stress fracture developed, or extended, during the days after the marathon, secondary to the 'leg-stretching' runs.

Long-distance athletes have a notoriously stoical attitude to pain and discomfort. This attitude, combined with the sacrifices made during months of training, contributes to the discounting of warning signals offered by the body, and results in the 'abnormal stresses to normal bone', and bone and organ failure.

If symptoms persist, despite conservative treatment and normal initial imaging, further imaging investigations are indicated.

\section{References}

1. Daffner RH, Pavlov H. Stress fractures: current concepts, AJR 1992; 158: 245-252.

2. Grainger RG, Allison D. Diagnostic Radiology. 3rd ed. Churchill Livingstone, 1999: 1574, 1901 1921.

3. Holder LE. Bone scintigraphy in skeletal trauma. Radiol Clin North Am 1994; 31: 739-781.

4. Holder LE, Schwartz C, Wernicke PG, Michael $\mathrm{RH}$. Radionuclide bone imaging in the early detection of fractures of the proximal femur (hip): multifactorial analysis. Radiology 1990; 152: 509-515. 